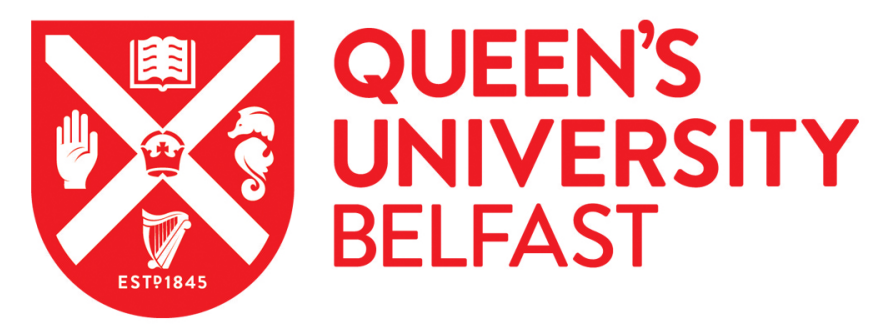

\title{
Ultra-Stable Plasmonic Colloidal Aggregates for Accurate and Reproducible Quantitative SE(R)RS in Protein-Rich Biomedia
}

Ye, Z., Li, C., Chen, Q., Xu, Y., \& Bell, S. (2019). Ultra-Stable Plasmonic Colloidal Aggregates for Accurate and Reproducible Quantitative SE(R)RS in Protein-Rich Biomedia. Angewandte Chemie (International ed. in English), 58(52), 19054. https://doi.org/10.1002/anie.201911608

Published in:

Angewandte Chemie (International ed. in English)

Document Version:

Peer reviewed version

Queen's University Belfast - Research Portal:

Link to publication record in Queen's University Belfast Research Portal

Publisher rights

Copyright 2019 Wiley. This work is made available online in accordance with the publisher's policies. Please refer to any applicable terms of use of the publisher.

\section{General rights}

Copyright for the publications made accessible via the Queen's University Belfast Research Portal is retained by the author(s) and / or other copyright owners and it is a condition of accessing these publications that users recognise and abide by the legal requirements associated with these rights.

Take down policy

The Research Portal is Queen's institutional repository that provides access to Queen's research output. Every effort has been made to ensure that content in the Research Portal does not infringe any person's rights, or applicable UK laws. If you discover content in the Research Portal that you believe breaches copyright or violates any law, please contact openaccess@qub.ac.uk. 


\title{
Ultra-Stable Plasmonic Colloidal Aggregates for Accurate and Reproducible Quantitative SE(R)RS in Protein-Rich Biomedia
}

\author{
Ziwei Ye, Chunchun Li, Qinglu Chen, Yikai $\mathrm{Xu}^{*}$ and Steven E. J. Bell*
}

\begin{abstract}
Au} / \mathrm{Ag}$ colloids aggregated with simple salts are amongst the most commonly used substrates in surfaceenhanced (resonance) Raman spectroscopy (SE(R)RS). However, salt-induced aggregation is a dynamic process, which means that $S E(R) R S$ enhancements vary with time and that measurements therefore need to be taken at a fixed time point, normally within a short time-window of a few minutes. Here, we present an emulsion templated method which allows formation of densely-packed quasi-spherical Au/Ag colloidal aggregates. Since the particles in the product aggregates retain their weakly adsorbed charged ligands and the ionic strength remains low these charged aggregates resist further aggregation while still providing intense $S E(R) R S$ enhancement which remains stable for days. This eliminates a major source of irreproducibility in conventional colloidal SE(R)RS measurements and paves the way for $S E(R) R S$ analysis in complex systems, such as proteinrich bio-solutions where conventional aggregated colloids fail.
\end{abstract}

\section{Introduction}

Plasmonic metal nanoparticles (NPs) display localized surface plasmon resonances (LSPR) which give them their unique optical properties and have been widely exploited in numerous applications, such as sensing, photo-therapy and catalysis..$^{[1]}$ Additionally, assembly of plasmonic NPs into secondary nanostructures induces near-field coupling of the surface plasmon among neighboring NPs, giving rise to new collective properties. ${ }^{[2]}$ A major field where plasmonic coupling is exploited is in surface enhanced (resonance) Raman spectroscopy $(\mathrm{SE}(\mathrm{R}) \mathrm{RS}$ ) where the intense electromagnetic fields in hot-spots created between neighboring Au/Ag NPs results in very large increase in the Raman scattering intensity. ${ }^{[3]}$ Raman signals of analytes within hot-spots can be enhanced by a factor of up to $10^{15}$, making it possible to provide fingerprint information on analytes even at single molecule level. ${ }^{[4]}$ Despite the large amounts of effort that have been devoted to preparing solid state SE(R)RS substrates, colloidal aggregates obtained by mixing $\mathrm{Au} / \mathrm{Ag}$ colloids with aggregating agents (typically high concentration salt solution) remain very widely used because they are inexpensive, easy to prepare and give very large enhancements. ${ }^{[4 b, 5]}$ However, despite this they are nevertheless often dismissed as irreproducible due to the randomness in the formation of colloidal aggregates. ${ }^{[6]}$ The underlying issue with salt-induced aggregation is that it is a dynamic process; the

[*] Z. Ye, C. Li, Q. Chen, Dr. Y. Xu, Prof. S. E. J. Bell School of Chemistry and Chemical Engineering, Queen's University of Belfast, University Road, Belfast, BT9 5AG Northern Ireland, United Kingdom

E-mail: yxu18@qub.ac.uk; s.bell@qub.ac.uk addition of salt reduces the electrostatic barrier that prevents charged particles from approaching each other. Without this barrier the interactions between particles are dominated by attractive Van der Waals forces so that the particles assemble into aggregates, which grow continuously right up to the point where they precipitate. This means that there is a fixed time window for SE(R)RS measurements which begins when the particles are sufficiently aggregated to form hot-spots and ends when the aggregates precipitate. Even within this window the enhancement may vary considerably. Moreover, the duration of this time window, along with the enhancement factor, changes when different types of aggregating agents or different concentrations of the same aggregating agent are used. ${ }^{[6 c, 7]}$ As a result, the time point at which $S E(R) R S$ measurement is taken needs to be carefully chosen so that particles are well aggregated but not yet precipitated. There have been reports on creating stable Au or Ag clusters or colloidosomes for SE(R)RS detection. ${ }^{[3 a, 8]}$ However, despite improvements in stability, the application of these clusters in $S E(R) R S$ is limited since their preparation requires surface modification of the particles or the use of surfactant.

Herein, we report a rapid, general and modifier-free approach to generate highly stable $\mathrm{SE}(\mathrm{R}) \mathrm{RS}$ active colloidal aggregates. Instead of using salt to induce aggregation, we exploit interfacial tension and charge screening provided by low concentrations of non-adsorbing amphiphilic ions to assemble particles onto the surface of oil-in-water emulsion droplets. These droplets then evaporate within minutes to reproducibly create aqueous suspensions of particle aggregates with quasi-spherical structures. Importantly, since the method does not require the surface of the particles to be modified, the constituent particles still carry their original charged ligands, which allows the aggregates to remain dispersed in solution through interaggregate electrostatic repulsion (Figure S1). Remarkably, the aggregates remain as individual assemblies for over a month without any further aggregation being detected by scanning electron microscopy (SEM) and dynamic light scattering (DLS) monitoring, so we have termed them stable plasmonic aggregates (SPAs). The fact that no additional surface modification to the NPs is required also means that the surfaces of SPAs remain chemically exposed for interaction with analytes in $S E(R) R S$ measurements. Therefore, using SPAs as enhancing substrates allows us to significantly extend the time window for colloidal SE(R)RS experiments from the usual minutes up to days with excellent reproducibility. In addition to the obvious advantages of removing the time dependence from analytical measurements, this stability also allows us to observe processes with low rates such as the adsorption of biomolecules (e.g. adenine) or drugs (e.g. phenytoin) onto enhancing surfaces from complex protein solutions. 


\section{Results and Discussion}

The main challenge in the preparation of SPAs is to drive the particles onto the surface of emulsion droplets without blocking the surface of the constituent NPs by adding strongly binding surface modifying ligands. We have previously reported that adding low concentrations $\left(\geq 10^{-7} \mathrm{M}\right)$ of amphiphilic "promoter" salts to provide counterions which sit in the oil phase allows charged NPs to assemble at oil-water liquid-liquid interfaces by screening the interparticle electrostatic repulsion. ${ }^{[9]}$ Here, the promoters are instead used to drive the NPs to the surface of the oil-in-water microemulsions.

Figure 1a illustrates the process for the formation of SPAs using mono-disperse ( $25 \pm 5 \mathrm{~nm}$ diameter) citrate-reduced Au colloid. To prepare Au SPAs, the Au colloid was sonicated with hexane to create oil-in-water emulsion droplets, with Au NPs assembled at the droplet interface (Figure 1a, step 1). This process was observed visually by a colour change of the colloid from ruby to purple immediately after sonication (Figure $1 \mathrm{~b}$ ). The evaporation of hexane over several minutes led to reduction of the interfacial surface area and the formation of tightly packed quasi-spherical NP-aggregates (Figure 1a, step 2), during which the colour of the colloid continued to evolve from purple to blue (Figure $1 \mathrm{~b}$ ). Figure 1c compares the extinction spectra of the parent $\mathrm{Au}$ colloid with that of the Au SPAs, where the broadened and redshifted LSPR shows the particles are strongly coupled. SEM images of the Au SPAs dried from solution (Figure 1e) show that they are quasi-spherical shells composed of closely packed $\mathrm{Au}$ NPs and range from ca. 50 to $300 \mathrm{~nm}$ in diameter. This correlates well with the DLS data shown in Figure 1d. The empty interior of SPAs can be clearly seen in an image of a damaged SPA, as shown in the inset of Figure 1e.

The promoter, tetrabutylammonium nitrate $\left(\mathrm{TBA}^{+} \mathrm{NO}_{3}{ }^{-}\right)$, induces NP self-assembly by providing interparticle charge screening while not absorbing directly onto the surface of Au NPs and since it is sparingly soluble in water it can re-dissolve into the aqueous phase after the evaporation of oil droplets. As a result, the SPAs bear the same surface ligands and surface charge as the parent colloid. This allows SPAs to remain separated from each other due to electrostatic repulsion provided by the charged ligands. ${ }^{[10]}$ Conversely, within the SPAs, Van der Waals forces between Au NPs hold them together to retain the overall structure. ${ }^{[10]}$ As a result, the SPAs are SE(R)RS-active in the same way as conventional aggregated colloids but are also sufficiently stable that they can be dispersed in water for extended periods and withstand centrifugal and even sonication forces. For example, Figure 1d shows that the size distributions of Au SPAs obtained from DLS, which did not change over 150 hours (see also corresponding SEM images, in Figure S2). Critical parameters such as particle size, particle concentration, sonication power, and sonication time were investigated to understand the mechanism of how these factors affect the stability and morphology of the product Au SPAs. It was found that an increase in particle size and a decrease in particle concentration led to the formation of broken shells or clusters, whereas the method showed more tolerance towards changes in sonication power and sonication time (see Figure S3).

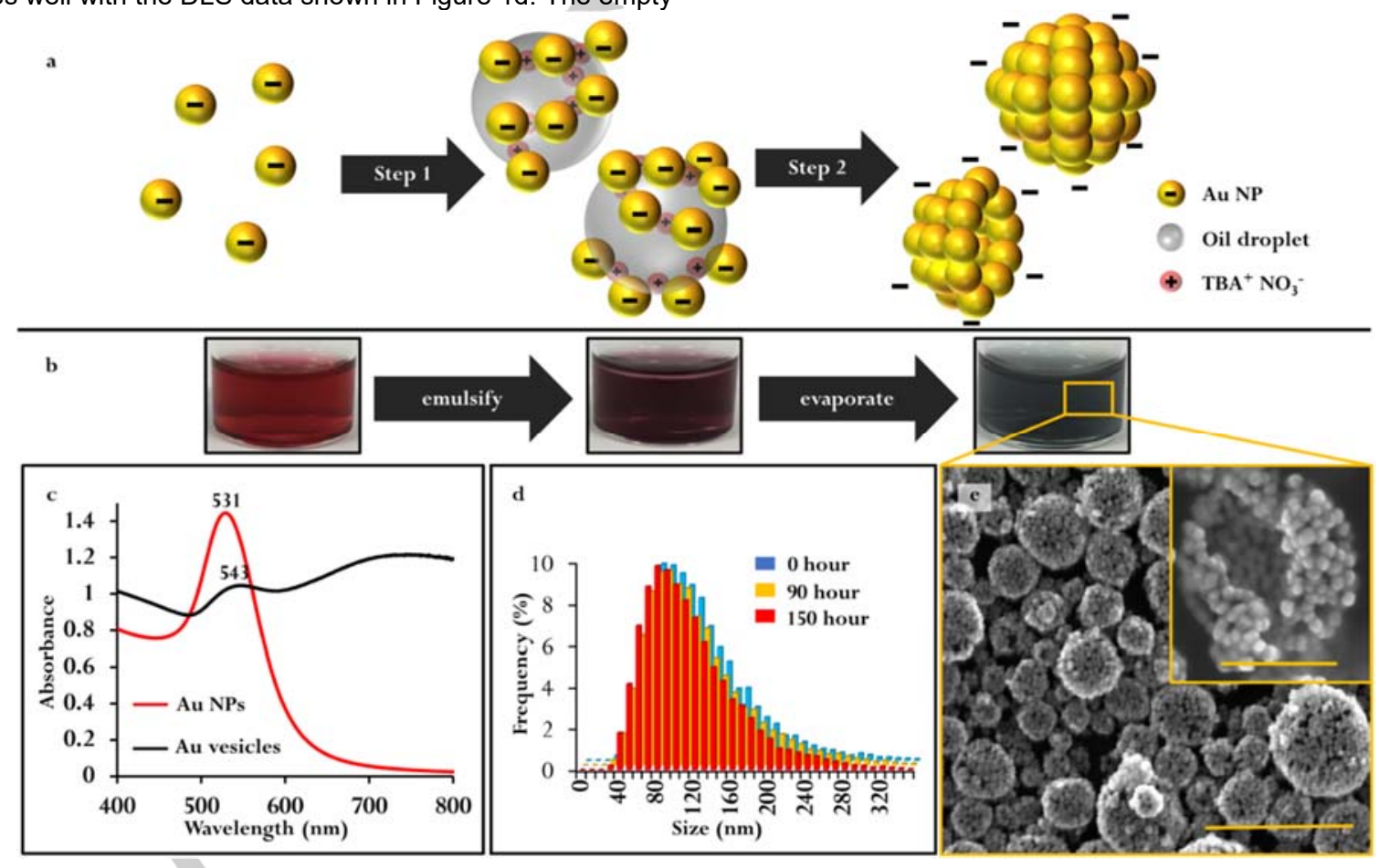

Figure 1. (a) Schematic illustration of the formation of Au SPAs: migration of Au NPs to the surface of oil droplets upon emulsification (Step 1); evaporation of oil droplets leading to the formation of SPAs (Step 2). (b) Optical images showing the colour of the Au colloid evolving throughout the self-assembly process. (c) UV/visible spectra of Au colloid before and after SPA formation. (d) Size distribution of the same batch of Au SPAs at different time points: immediately after formation (blue bar); after 90 hours (yellow bar); after 150 hours (red bar). (e) SEM image of Au SPAs. Inset shows a single Au SPA with a cracked particle shell. Scale bars in (e) and its inset correspond to $500 \mathrm{~nm}$ and $250 \mathrm{~nm}$, respectively. 
Here we also show SPAs prepared from the citrate reduced $\mathrm{Ag}$ particles which are very poly-disperse to demonstrate the generality of the method. Similar to SPAs formed from monodisperese Au NPs, these highly irregular Ag particles formed quasi-spherical aggregates which remained stable in solution for $>150$ hours (Figure S4). As expected, the Ag SPAs gave larger $\mathrm{SE}(\mathrm{R}) \mathrm{RS}$ enhancements than the SPAs formed using $25 \pm 5 \mathrm{~nm}$ diameter Au NPs so further investigations concentrated on $\mathrm{Ag}$ SPAs.

As shown in Figure 2a, the normalized $S E(R) R S$ spectra of $A g$ SPAs and of the parent colloid aggregated with $\mathrm{MgSO}_{4}$ are identical and show the characteristic bands of the surface citrate layer $\left(1398,1024,950,805 \mathrm{~cm}^{-1}\right)$ which both carry. ${ }^{[11]}$ The additional bands at 1988 and $2107 \mathrm{~cm}^{-1}$ within the SE(R)RS signals of Ag SPA possibly arise from a small amount of weakly adsorbed $\mathrm{CN}^{-}$from the environment and did not have any notable effect on the SE(R)RS properties of the Ag SPAs. ${ }^{[12,13]}$ The SERS data give direct evidence that the citrate surface layer is retained in the SPAs and that the added $\mathrm{TBA}^{+} \mathrm{NO}_{3}$ promoter does not directly absorb onto the Ag NPs. The citrate band at $1024 \mathrm{~cm}^{-1}$ can be used as a convenient internal standard for measuring reproducibility of the enhancement given by the SPAs. Figure $2 b$ shows the absolute $S E(R) R S$ intensity of citrate band acquired from 10 different Ag SPA samples which have a relative standard deviation $<11 \%$. To investigate the signal stability of Ag SPAs, we labelled 4 different Ag SPA samples with the same amount of crystal violet (CV), a dye commonly used in SE(R)RS analysis, and then recorded their signal intensity every 24 hours up to 144 hours. The strong CV band at $1173 \mathrm{~cm}^{-1}$ was used as the reference band. The data in Figure $2 \mathrm{c}$ (red bars) shows that the relative standard deviation in the intensity of the signals from different samples recorded at each time point varied between $7-20 \%$. It is worth noting that the average signal deviation after 144 hours was ca. $8.5 \%$, which was slightly lower than that at the beginning of the experiment. A t-test of the data confirmed that this difference was not statistically significant (see $\mathrm{SI}$ ). This experiment shows that the time window for making measurements after the addition of sample to SPAs colloid is at least several days. To explore the possibility of using pre-synthesized SPAs as SE(R)RS enhancing substrates, the SE(R)RS intensity of the CV band at $1173 \mathrm{~cm}^{-1}$ was also studied using Ag SPAs which have been stored for different lengths of time (Figure $2 \mathrm{c}$ orange bars). For SPAs that have been stored between 0-144 hours, the deviation in $S E(R) R S$ signal intensity was only $12.2 \%$, which shows that the morphology of $\mathrm{Ag}$ SPAs remained unchanged and that the hot-spots in the Ag SPAs remained fully accessible even several days post-fabrication. Similar to large colloidal NPs, SPAs exhibit weaker Brownian motion and are more affected by gravity than individual particles. As a result, they will slowly settle to the bottom of the vessel if left undisturbed after several days. However, even under such circumstances the electrostatic repulsion between SPAs prevents further aggregation and allows SPAs to be re-dispersed without any obvious loss to their SE(R)RS enhancing abilities (Figure S5).

To further evaluate the capabilities of Ag SPAs as a SE(R)RS substrate, measurements were performed with Ag SPAs and CV at different concentrations. As shown in Figure 2e, SE(R)RS signals of CV could be observed down to $10^{-10} \mathrm{M}$ and showed a steady increase up to $10^{-6} \mathrm{M}$. By measuring the intensity of the peak for $\mathrm{CV}$ at $1173 \mathrm{~cm}^{-1}$, the linear quantitative range was determined to be $10^{-10}-10^{-7} \mathrm{M}$ with a $\mathrm{R}^{2}$ value of 0.995 . Moreover, the Analytical Enhancement Factor (AEF) for the SPAs were calculated to be ca. $1.4 \times 10^{5}$, which is amongst the highest level of signal enhancement for colloidal SE(R)RS substrates (Figure S6). ${ }^{[14]}$ It should be noted that this high AEF value was achieved with SPAs fabricated from the most common citrate reduced $\mathrm{Ag}$ colloid. In principle, our method can be readily extended to fabricate SPAs from various types of colloidal NPs regardless of their morphology and material composition. This means that SPAs with even stronger SE(R)RS enhancement can potentially be fabricated using more plasmonically active colloidal building blocks.

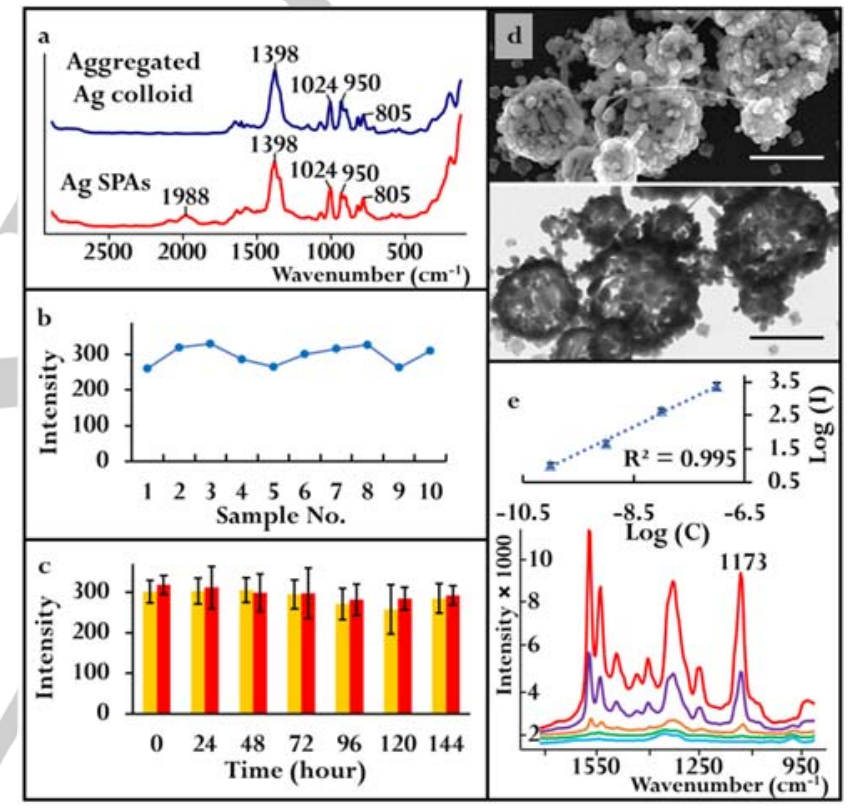

Figure 2. (a) $\mathrm{SE}(\mathrm{R}) \mathrm{RS}$ spectra of $\mathrm{MgSO}_{4}$ aggregated $\mathrm{Ag}$ colloid (i) and $\mathrm{Ag}$ SPAs (ii). The intensities of the spectra ( $y$ axis) have been rescaled for illustration. (b) $\mathrm{SE}(\mathrm{R}) \mathrm{RS}$ intensity of blank $\mathrm{Ag}$ SPAs collected from 10 independently prepared samples. (c) $S E(R) R S$ intensity of $C V$ labelled $\mathrm{Ag}$ SPAs (red bars) collected every 24 hours over a total of 144 hours; SE(R)RS intensity of CV collected using SPAs which have been stored for different lengths of time after synthesis (orange bar). The intensity of $\mathrm{CV}$ was measured using the peak at $1173 \mathrm{~cm}^{-1}$ (d) SEM images of Ag SPAs imaged using secondary electron detector (top) and back scattering detector (bottom). Scale bars in (d) correspond to $500 \mathrm{~nm}$. (e) SE(R)RS spectra of CV with the concentration ranging from $10^{-10} \mathrm{M}$ to $10^{-6} \mathrm{M}$. The full range spectra are shown in Figure S7. Inset shows calibration curve for concentration versus intensity.

SE(R)RS has recently emerged as a non-invasive and ultrasensitive analytical technique for rapid detection of biologically active molecules and metabolites in biological media. ${ }^{[15]}$ However, body fluids such as plasma typically contain high concentrations of proteins, which adsorb strongly to the surface of colloidal NPs to form coronas. This not only prevents analyte molecules from adsorbing onto the enhancing particles but also stops the colloid from aggregating and forming 
SE(R)RS active hot-spots. ${ }^{[16]}$ As such, SE(R)RS analysis of samples in biological media with colloidal substrates has typically required pre-treatment steps to first remove proteins from the sample, which is inconvenient and time-consuming. ${ }^{[17]}$ Alternatively, it is also possible to first aggregate the colloid with salt to form hot-spots before adding in the sample. However, this narrows the window for SE(R)RS analysis and adds to the irreproducibility of the measurements. ${ }^{[18]}$ To test the potential of SPAs for SE(R)RS analysis in biological media, samples of adenine dissolved in $3.3 \%$ albumin solution were used to provide a simple biologically-relevant test molecule in a solution whose protein content matched that of human serum. Even at much lower protein concentrations conventional colloids fail to give any $S E(R) R S$ signals since they cannot be aggregated even with the addition of high concentrations of salt. Conversely, since the SPAs carry stable pre-formed plasmonic hot-spots, using Ag SPAs as the enhancing substrate allowed adenine to be detected down to $0.01 \mu \mathrm{M}$ despite the large amount of protein present (Figure 3d). As mentioned above, it is possible to aggregate the colloid with salt before addition of sample. However, the maximum signal of adenine was observed after 30 mins of sample incubation time, which is well beyond the detection time window for salt-aggregated colloid. The excellent stability of SPAs also meant that they can be used to provide valuable $S E(R) R S$ information of the adsorption kinetics of adenine in protein solution. As shown in Figure $3 \mathrm{a}$, when $\mathrm{Ag}$ SPAs are mixed with adenine/protein solution, initially only citrate bands are observed but the characteristic adenine peak at $731 \mathrm{~cm}^{-1}$ gradually grows over time. Since the rise in adenine signal is accompanied by a fall in citrate signals it is clearly associated with gradual displacement of citrate in the hot-spots by adenine, rather than some form of time-dependent change in the structure of the SPAs. Figure $3 \mathrm{c}$ shows the signal growth kinetics at adenine concentrations of $0.01 \mu \mathrm{M}, 0.05 \mu \mathrm{M}$ and 0.1 $\mu \mathrm{M}$, respectively. For the $0.1 \mu \mathrm{M}$ sample the growth follows first order kinetics with $k=3.8 \times 10^{-4} \mathrm{~s}^{-1}$, as shown in Figure S8. For the lower concentrations the signals are too small to determine reliable rate constants. However, for all the concentrations tested, the SE(R)RS intensity of adenine took tens of minutes to reach a maximum in albumin solution, which contrasted strongly with detection of adenine in DDI water where the SE(R)RS intensity of adenine reached maximum immediately after mixing the sample with SPAs. This suggests that the slow adsorption kinetics observed for adenine in the presence of protein is not due to the diffusion rate of adenine in bulk solution but rather due to the protein adsorbing onto the outer surface areas of SPAs and thus hindering the diffusion of adenine into SE(R)RS active hot-spots between NPs. As shown in Figure 3c, the adsorption kinetics of adenine in protein solution varies significantly at different concentrations but, as the quantitative data in Figure $3 \mathrm{~d}$ show, the SPAs are sufficiently stable to allow the effect of non-equilibrium binding to be minimised by allowing long incubation times. This is not possible with conventional colloidal substrates since they cannot remain stable long enough to reach adsorption equilibrium. It is worth noting that Ag SPAs' ability to detect analyte molecules in protein-rich bio-solutions is not limited to adenine. As shown in Figure S9, Ag SPAs can also be readily used to detect important drug molecules, such as phenytoin in albumin rich solution.

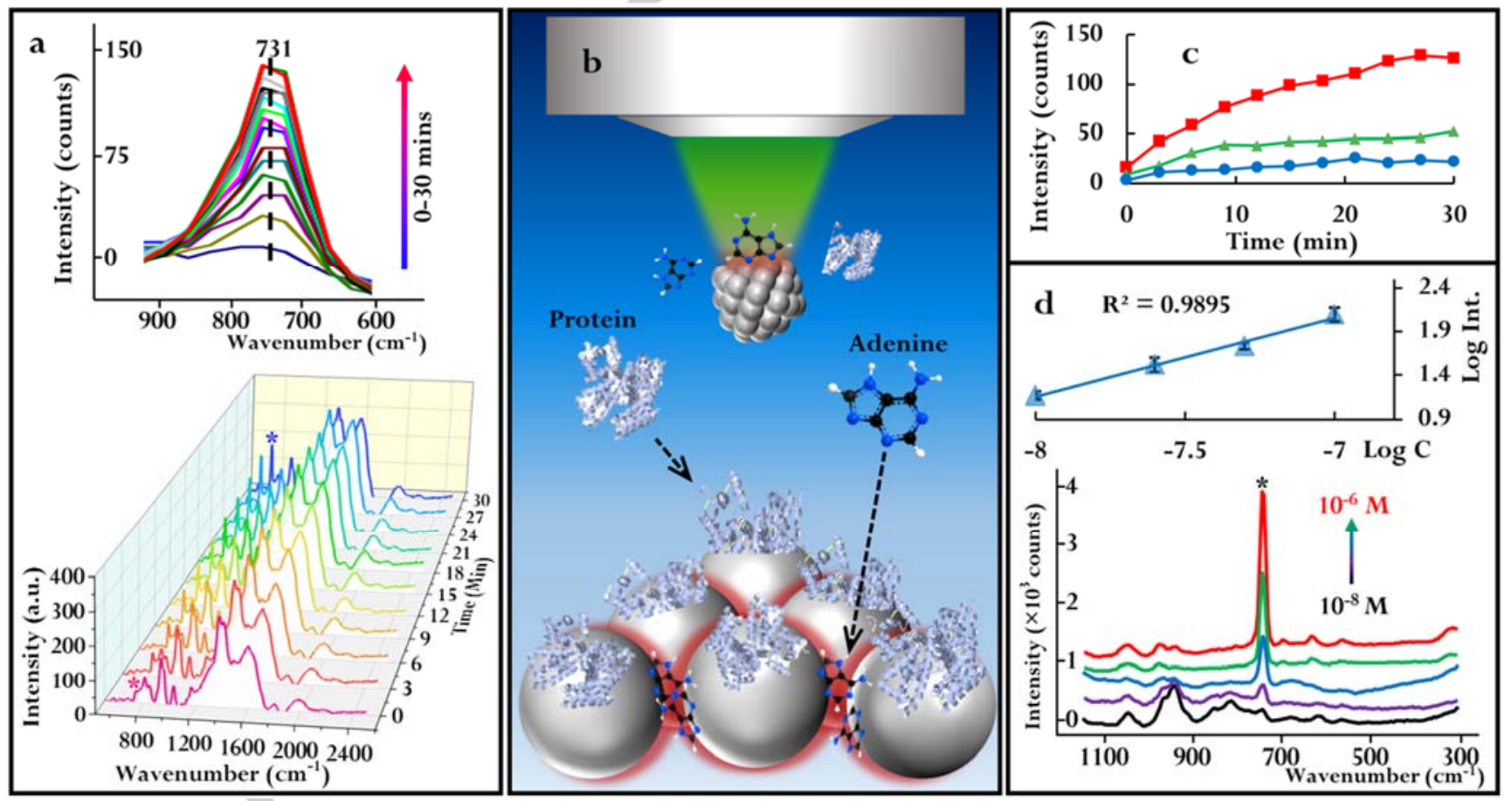

Figure 3. (a) Time evolution of SE(R)RS spectra after mixing Ag SPAs with adenine/albumin solution containing $0.1 \mu \mathrm{M}$ of adenine and $3.3 \%$ albumin. Inset on the top shows the growth of the adenine peak at $731 \mathrm{~cm}^{-1}$ over 30 mins. (b) Schematic representation of adenine diffusion into the hot-spots of SPAs while protein only adsorbs to the outer-surface. (c) Growth of adenine peak $\left(731 \mathrm{~cm}^{-1}\right.$ ) at various adenine concentrations versus time: $0.1 \mu \mathrm{M}$ (red line), $0.05 \mu \mathrm{M}$ (green line) and $0.01 \mu \mathrm{M}$ (yellow line). (d) SE(R)RS spectra of adenine at different concentrations dissolved in protein-rich solution acquired with Ag SPAs after 30 min incubation time along with the corresponding calibration curve for adenine concentration versus $S E(R) R S$ signal intensity $\left(731 \mathrm{~cm}^{-1}\right)$. 
Interestingly, for the Ag SPAs the absolute signal intensity of the adenine in the presence of albumin was consistently ca. $3 x$ higher than in the absence of adenine (Figure S10). This was unexpected since protein is known to compete with adenine for enhancing surface. One plausible explanation is that due to steric hindrance, albumin can only adsorb onto the lessenhancing open surface of the SPAs, which drives the small adenine molecules into the much more strongly enhancing SE(R)RS hot-spots between NPs (Figure $3 b$ ).

\section{Conclusion}

In conclusion, we have demonstrated an emulsion templated method to reproducibly produce ultra-stable $S E(R) R S$ active colloidal aggregates with quasi-spherical structures. Since our method only requires the use of low concentrations of nonadsorbing promoter ions to provide charge-screening the NPs' surfaces are unchanged. As a result, the product aggregates can remain stable for days due to inter-aggregate electrostatic repulsion provided by the charged capping agents on the surface of the constituent NPs. This dramatically contrasts with conventional aggregated colloids which are extremely unstable and typically give enhancements which change over time and decay completely within minutes. The fact that no surface modification is performed also means that the surfaces of the NPs are chemically exposed so that they are free to interact with weakly adsorbing analytes. All the above properties combine to make these SPAs superior to conventional salt-induced aggregates for standard SE(R)RS measurements since they remove uncertainty associated with time-dependent changes. In addition, they also allow more challenging samples with slow binding kinetics in bio-media to be analysed, which paves the way for various important SERS applications, such as long-term in-vivo monitoring of bio-reactions or drug-release.

\section{Experimental Section}

Silver nitrate, tetrachloroauric (III) acid trihydrate, trisodium citrate dihydrate, tetrabutylammonium nitrate, hexane, adenine, phenytoin, magnesium sulphate, albumin and crystal violet were purchased from Sigma Aldrich. All experiments used low TOC (<3.0 ppb) $18.2 \mathrm{M} \Omega \mathrm{cm}$ distilled deionized (DDI) water.

Citrate-reduced Ag colloids were prepared using the Lee and Meisel method. ${ }^{[19]}$ The typical optical density of Ag colloids was 1.6 and the concentration of $\mathrm{Ag}$ colloids was measured to be $5.8 \times 10^{11}$ particles $/ \mathrm{mL}$. Citrate-reduced Au colloids were prepared using the Frens method with slight modifications. ${ }^{[9 b]}$ Briefly, $50 \mathrm{mg}$ of tetrachloroauric (III) acid trihydrate dissolved in $50 \mathrm{~mL}$ of DDI water in a round bottom flask was heated to boiling at reflux under vigorous stirring. Then $8 \mathrm{~mL}$ of aqueous sodium citrate $(1 \mathrm{wt} \%)$ solution was added into the reaction mixture all at once. The mixture was kept boiling for another 30 mins before being cooled naturally to room temperature. The product Au colloids contained mono disperse Au NPs which were $25 \pm 5 \mathrm{~nm}$ in diameter. The typical optical density of Au colloids was 1.4 and the concentration of Au colloids was measured to be $2 \times 10^{12}$ particles $/ \mathrm{mL}$.
For SPA preparation, a Soniprep 150 Ultrasonic Disintegrator was used for emulsification. In a typical synthesis, $1 \mathrm{~mL}(3 \mathrm{~mL})$ of $\mathrm{Au}(\mathrm{Ag})$ colloid was first diluted to $4 \mathrm{~mL}(2 \mathrm{~mL})$ with DDI water. This colloid was then emulsified with $0.3 \mathrm{~mL}$ of hexane, and $50 \mu \mathrm{L}$ of $10^{-3} \mathrm{M} \mathrm{TBA}^{+} \mathrm{NO}_{3}^{-}$using a power of 65 watts for $12 \pm 3 \mathrm{~s}(20 \pm 5 \mathrm{~s})$. The standard probe position for emulsification is shown in Figure S11. After emulsification, the mixture was left for half an hour under room temperature to allow complete evaporation of hexane to produce stable SPA colloids, which were stored at room temperature and typically used within 2 weeks.

For $\mathrm{SE}(\mathrm{R}) \mathrm{RS}$ measurements conventional aggregated Ag colloid samples were prepared by mixing $20 \mu \mathrm{L}$ of $1 \mathrm{M} \mathrm{MgSO}_{4}$ solution and 20 $\mu \mathrm{L}$ of analyte solution with $200 \mu \mathrm{L}$ of $\mathrm{Ag}$ colloid. Ag SPA samples were prepared by mixing $20 \mu \mathrm{L}$ of analyte solution with $200 \mu \mathrm{L}$ of Ag SPAs. SERS measurements were performed using an WITec Alpha $300 R$ Confocal Raman Microscope equipped with a $532 \mathrm{~nm}$ diode laser with an output of $60 \mathrm{~mW}, \mathrm{a} \times 10$ lens with a spot diameter of $1.6 \mu \mathrm{m}$ and by focusing the laser beam on the air-water interface of the solutions and using a total accumulation time of $50 \mathrm{~s}$.

\section{Acknowledgements}

Z. Ye, Y. Xu and C. Li were funded by the University Special Research Scholarship (Q.U.B.). We thank Prof. Eric V. Anslyn for his valuable advice on the manuscript.

Keywords: aggregates, interfaces, colloidosomes, SE(R)RS, self-assembly

[1] a) Y. Liu, J. He, K. Yang, C. Yi, Y. Liu, L. Nie, N. M. Khashab, X. Chen, Z. Nie, Angew. Chem. Int. Ed. 2015, 54, 15809-15812; Angew. Chem. 2015, 127, 1603516038; b) Y. Zhang, S. He, W. Guo, Y. Hu, J. Huang, J. R. Mulcahy, W. D. Wei, Chem. Rev. 2018, 118, 29272954; c) Y. Zhang, J. Qian, D. Wang, S. He, Angew. Chem. Int. Ed. 2013, 52, 1148-1151; Angew. Chem. 2013, 125, 1186-1189.

[2] S. K. Ghosh, T. Pal, Chem. Rev. 2007, 107, 4797-4862.

[3] a) G. C. Phan-Quang, H. K. Lee, I. Y. Phang, X. Y. Ling, Angew. Chem. Int. Ed. 2015, 54, 9691-9695; Angew. Chem. 2015, 127, 9827-9831; b) Y. Xu, Z. Ye, C. Li, H. McCabe, J. Kelly, S. E. J. Bell, Appl. Mater. Today 2018, 13, 352-358; c) Matricardi, C. Hanske, J. L. Garcia-Pomar, J. Langer, A. Mihi, L. M. Liz-Marzán, ACS Nano 2018, 12, 8531-8539.

[4] a) S. Nie, S. R. Emory, Science 1997, 275, 1102-1105; b) K. Kneipp, Y. Wang, H. Kneipp, L. T. Perelman, I. Itzkan, R. R. Dasari, M. S. Feld, Phys. Rev. Lett. 1997, 78, 1667-1670.

[5] a) Y. Gao, L. Li, X. Zhang, X. Wang, W. Ji, J. Zhao, Y. Ozaki, Chem. Commun. 2019, 55, 2146-2149; b) Z. Ye, C. Li, Y. Xu, S. E. J. Bell, Analyst 2019, 144, 448-453; c) Y. Lai, S. Schlücker, Y. Wang, Anal. Bioanal. Chem. 2018, 410, 5993-6000.

[6] a) X.-M. Lin, Y. Cui, Y.-H. Xu, B. Ren, Z.-Q. Tian, Anal. Bioanal. Chem. 2009, 394, 1729-1745; b) N. M. Kovalchuk, V. M. Starov, Adv. Colloid Interface Sci. 2012, 179-182, 99-106; c) R. Tantra, R. J. C. Brown, M. J. T. Milton, J. Raman Spectrosc. 2007, 38, 1469-1479.

[7] A. M. Schwartzberg, C. D. Grant, A. Wolcott, C. E. Talley, T. R. Huser, R. Bogomolni, J. Z. Zhang, J. Phys. Chem. B 2004, 108, 19191-19197. 
[8] Z. Han, H. Liu, B. Wang, S. Weng, L. Yang, J. Liu, Anal. Chem. 2015, 87, 4821-4828.

[9] a) Y. Xu, M. P. Konrad, W. W. Y. Lee, Z. Ye, S. E. J. Bell, Nano Lett. 2016, 16, 5255-5260; b) Z. Ye, C. Li, N. Skillen, Y. Xu, H. McCabe, J. Kelly, P. Robertson, S. E. J. Bell, Appl. Mater. Today 2019, 15, 398-404.

[10] V. N. Manoharan, M. T. Elsesser, D. J. Pine, Science 2003, 301, 483-487.

[11] C. H. Munro, W. E. Smith, M. Garner, J. Clarkson, P. C. White, Langmuir 1995, 11, 3712-3720.

[12] S. Sánchez-Cortés, J. V. García-Ramos, J. Raman Spectrosc.1998, 29, 365-371.

[13] M. Fleischmann, Z. Q. Tian, L. J. Li, J. Electroanal. Chem. 1987, 217, 397-410.

[14] a) E. C. Le Ru, E. Blackie, M. Meyer, P. G. Etchegoin, J. Phys. Chem. C 2007, 111, 13794-13803; b) L. Scarabelli, M. Coronado-Puchau, J. J. Giner-Casares, J. Langer, L. M. Liz-Marzán, ACS Nano 2014, 8, 58335842.

[15] a) S. Laing, K. Gracie, K. Faulds, Chem. Soc. Rev. 2016, 45, 1901-1918; b) A. F. Robson, T. R. Hupp, F. Lickiss, K. L. Ball, K. Faulds, D. Graham, Proc. Natl. Acad. Sci. U.S.A. 2012, 109, 8073-8078; c) D. CiallaMay, X.-S. Zheng, K. Weber, J. Popp, Chem. Soc. Rev. 2017, 46, 3945-3961.

[16] a) J. Mosquera, I. García, M. Henriksen-Lacey, G. González-Rubio, L. M. Liz-Marzán, Chem. Mater. 2019, 31, 57-61; b) B. Pelaz, G. Charron, C. Pfeiffer, Y. Zhao, J. M. de la Fuente, X-J. Liang, W. J. Parak, P. del Pino, Small 2013, 9, 1573-1584.

[17] a) N. E. Markina, I. Y. Goryacheva, A. V. Markin, Anal. Bioanal. Chem. 2018, 410, 2221-2227; b) A. Subaihi, D. K. Trivedi, K. A. Hollywood, J. Bluett, Y. Xu, H. Muhamadali, D. I. Ellis, R. Goodacre, Anal. Chem. 2017, 89, 4702-6709.

[18] E. Dumont, C. De Bleye, J. Cailletaud, P.-Y. Sacré, P.B. Van Lerberghe, B. Rogister, G. A. Rance, J. W. Aylott, Ph. Hubert, E. Ziemons, Talanta 2018, 185, 816.

[19] P. C. Lee, D. Melsel, J. Phys. Chem. 1982, 86, 33913395. 


\section{Entry for the Table of Contents}

Layout 1:

\section{RESEARCH ARTICLE}

Using emulsion-templated selfassembly allows reproducible and surface-modification-free formation of quasi-spherical colloidal aggregates which remain stable for days. The high stability and strong plasmonic enhancement of these aggregates allow accurate and quantitative $\mathrm{SE}(\mathrm{R}) \mathrm{RS}$ detection of weakly adsorbing analytes in protein-rich biomedia to be achieved.
Ziwei Ye, Chunchun Li, Qinglu Chen,

Yikai X $u^{\star}$, Steven E. J. Bell*

Page No. - Page No.

Ultra-Stable Plasmonic Colloidal

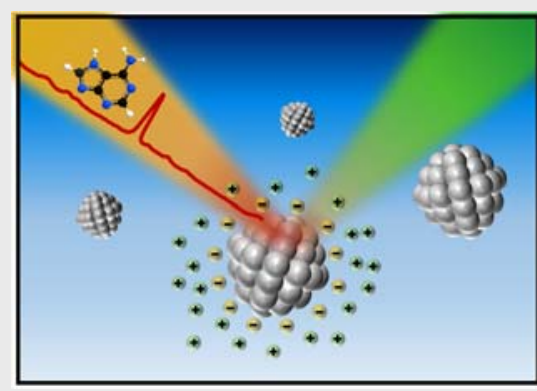

Aggregates for Accurate and Reproducible Quantitative SE(R)RS in Protein-Rich Biomedia 